\title{
WRINKLES IN SQUARE MEMBRANES
}

\author{
Yew Wei Wong and Sergio Pellegrino \\ Department of Engineering, University of Cambridge, Trumpington Street, Cambridge CB2 IPZ, UK
}

Summary This paper investigates the wrinkling of square isotropic membranes subject to coplanar pairs of equal and opposite corner forces. Two wrinkling regimes are observed experimentally and then reproduced by means of finite-element simulations. A general methodology is presented for making preliminary analytical estimates of wrinkle patterns and average wrinkle amplitudes and wavelengths, while also gaining physical insight into the wrinkling of membranes.

\section{INTRODUCTION}

Thin, prestressed membranes will be required for the next generation of spacecraft, to provide deployable mirror surfaces, solar collectors, sunshields, solar sails, etc. Some applications require membranes that are perfectly smooth in their operational configuration; other applications can tolerate membranes that are wrinkled with a known, small deviation from the nominal shape. Because the design of membrane structures with biaxial pretension, which would have a smooth surface, significantly increases the overall complexity of the structure, for those applications in which sufficiently small wrinkles are acceptable, engineers need to estimate the extent, wavelength and amplitude of the wrinkles.

The wrinkling of membranes has attracted much interest in the past, starting from the development of the tension field theory [1]. Simpler formulations and extensions of this theory were later proposed [2-7]. All of these formulations, with accompanying numerical solutions [8,9], model the membrane as a no-compression, two-dimensional continuum with negligible bending stiffness. However, the bending stiffness of the membrane cannot be neglected in the present case.

The problem considered in this paper is finding the characteristics of the wrinkles in a uniform elastic square membrane (which is a simple model of a square solar sail) of side length $L$ and thickness $t$ that is prestressed by two pairs of equal and opposite concentrated forces, $T_{1}$ and $T_{2}$. These forces are uniformly distributed over a small length $d$ at the corners. This membrane is isotropic with Young's Modulus $E$ and Poisson's ratio $\nu$; it is also initially stress free and perfectly flat (before the application of the corner forces).

\section{EXPERIMENTAL OBSERVATIONS}

Figure 1 shows photographs of the wrinkle patterns in a Kapton membrane with $L=500 \mathrm{~mm}, t=0.025 \mathrm{~mm}$, and $d=25 \mathrm{~mm}$. For symmetric loading $\left(T_{1}=T_{2}\right)$, Figure 1(a), the wrinkle pattern is fairly symmetric, with wrinkles radiating from each corner; the central region is free of wrinkles. For $T_{1} / T_{2}=2$ the wrinkles grow in amplitude but remain concentrated at the corners. Then, for $T_{1} / T_{2}=3$ a large diagonal wrinkle becomes visible, whose amplitude grows further for $T_{1} / T_{2}=4$.

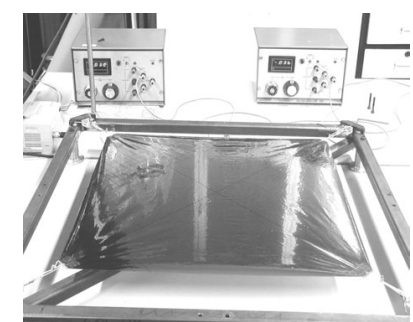

(a)

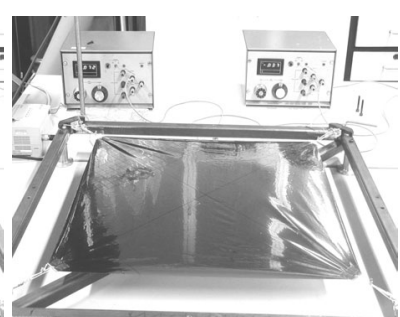

(b)

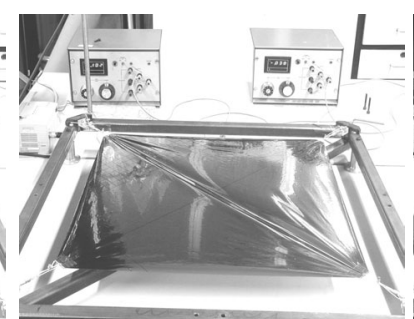

(c)

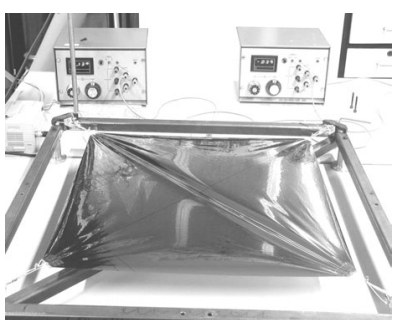

(d)

Figure 1. Wrinkled shapes for $T_{1}$ equal to (a) $5 \mathrm{~N}$, (b) $10 \mathrm{~N}$, (c) $15 \mathrm{~N}$, and (d) $20 \mathrm{~N} ; T_{2}=5 \mathrm{~N}$ in all cases.

\section{ANALYTICAL APPROACH}

Our analytical approach is in four parts, as follows. First, we propose a two-dimensional stress field that involves no compression anywhere in the membrane; the regions where the minor principal stress is zero are then assumed to be wrinkled, and the wrinkles are assumed to be along the major principal stress directions. Ideally, both equilibrium and compatibility should be satisfied everywhere by the proposed stress field, but analytical solutions in closed-form —obtained by tension field theory - exist only for very simple boundary conditions. We have recently shown [10] that a carefully chosen, simple stress field that satisfies only equilibrium can provide quick solutions that are useful for preliminary design. More accurate stress fields can be obtained from a two-dimensional stress analysis with membrane finite elements.

Second, we note that the bending stiffness of the membrane is finite, although small, and hence a compressive stress will exist in the direction perpendicular to the wrinkles. Because of its small magnitude, this stress was neglected in our previous analysis of the stress field. We assume that this compressive stress varies only with the wavelength of the wrinkles 
and set it equal to the critical buckling stress of a thin plate in uniaxial compression. Thus, the stress across the wrinkles is a known function of the wrinkle wavelength.

Third, we enforce equilibrium in the out-of-plane direction. Since the stress distribution is known, except for the wrinkle wavelength, a single equation of equilibrium will determine the wrinkle wavelength.

Fourth, the wrinkle amplitudes are estimated by considering the total strain in the membrane as the sum of two components, a material strain and a wrinkling strain.

\section{RESULTS}

We consider two kinds of simple equilibrium stress fields, one for symmetric loading and asymmetric loading with $T_{1} / T_{2} \leq 1 /(\sqrt{2}-1)$, and one for $T_{1} / T_{2} \geq 1 /(\sqrt{2}-1)$. The first field corresponds to fan-shaped corner wrinkles, which are represented in terms of sine functions. Analytical expressions are then derived for the number of corner wrinkles and their average maximum amplitude. The second field provides, in addition, a large diagonal wrinkle.

For example, we have obtained the following expressions for the wavelength and amplitude of this central wrinkle

$$
\begin{gathered}
\lambda=\sqrt[4]{\frac{2 \pi^{2} R_{1}\left(R_{1}-r_{1}\right)^{2} E t^{3} \sin \theta_{1}}{3\left(1-\nu^{2}\right) T_{1}}} \\
A=\frac{2 \sqrt{\lambda\left(\delta_{1}+\delta_{2}\right)}}{\pi}
\end{gathered}
$$

Here $\delta_{1}$ and $\delta_{2}$ are the radial displacements of the corners loaded by $T_{1}$ and $T_{2}$, respectively. They can be estimated from

$$
\delta_{i} \approx \frac{T_{i}}{2 E t \sin ^{2} \theta_{i}}\left[\theta_{i} \ln \left|\frac{R_{i}}{r_{1}}\right|+(1-\nu)\left(\frac{A}{R_{i}^{2}}+\theta_{i}-\frac{1}{2} \tan \theta_{i}\right)\right] \quad i=1,2
$$

More refined estimates can be obtained from two-dimensional finite-element analysis.

\section{DISCUSSION}

For the load case $T_{1} / T_{2}=4$, Table 1 compares the analytical predictions for the large diagonal wrinkle at the centre of the membrane, for two different membrane thicknesses, with predictions from ABAQUS and experimental measurements. The wavelength predictions are very close. The wrinkle amplitudes have been estimated first using only analytical solutions, Equation 2 with $\delta_{1}$ and $\delta_{2}$ from Equation 3, and second using Equation 2 but with corner deflections from a 2-D analysis. We can see that the fully analytical estimates are up to $88 \%$ higher than those measured experimentally, but if corner displacements from a 2D finite-element analysis are used, the estimates are only $48 \%$ and $16 \%$ higher than the measurements.

Finite element analysis using thin shell elements is able to replicate real physical experimentation with an accuracy typically better than $10 \%$ on amplitude. However, a very fine mesh had to be used to resolve the small corner wrinkles.

\section{References}

[1] H. Wagner. Flat sheet metal girder with very thin metal web, Zeitschrift für Flugtechnik Motorlurftschiffahrt. 20, 200-207, 227-233, 256-262, 279-284 (1929)

[2] E. Reissner. On tension field theory. Proc. 5th Int. Cong. Appl. Mech., 88-92 (1938).

[3] M. Stein and J.M. Hedgepeth. Analysis of Partly Wrinkled Membranes. NASA Langley Research Center, NASA TN D-813 (1961).

[4] E.H. Mansfield. Tension field theory a new approach which shows its duality with inextensional theory. Proc. 12th Int. Cong. Appl. Mech., 305-320 (1969).

[5] A.C. Pipkin. The relaxed energy density for isotropic elastic membranes. IMA J. Appl. Math., 36 85-99 (1986).

[6] M. Epstein and M.A. Forcinito. Anisotropic membrane wrinkling: theory and analysis. Int. J. Solids Structures. 38, 5253-5272 (2001).

[7] C. Jenkins and J.W. Leonard. Nonlinear dynamic response of membranes: State of the art. ASME Appl. Mech. Reviews, 44, 319-328 (1991).

[8] A. Adler. Finite Element Approaches for Static and Dynamic Analysis of Partially Wrinkled Membrane Structures. Ph.D. Dissertation, University of Colorado at Boulder (2000).

[9] Y.W. Wong and S. Pellegrino. Amplitude of wrinkles in thin membranes. In: New Approaches to Structural Mechanics, Shells and Biological Structures, edited by H.R. Drew and S. Pellegrino. Kluwer Academic Publishers, 257-270 (2002).

\begin{tabular}{|c|ccc|cccc|}
\hline$t$ & \multicolumn{3}{|c|}{$\lambda(\mathrm{mm})$} & \multicolumn{4}{c|}{$A(\mathrm{~mm})$} \\
$(\mathrm{mm})$ & Eq. 1 & Exp. & FE & Eqs 2, 3 & Eq. 2+FE & Exp. & FE \\
\hline 0.025 & 24.6 & 23.8 & 22.3 & 3.5 & 2.8 & 1.9 & 2.0 \\
0.050 & 41.3 & 33.9 & 35.6 & 3.2 & 2.1 & 1.8 & 1.6 \\
\hline
\end{tabular}

Table 1. Diagonal wrinkle half-wavelengths and amplitudes for $T_{1} / T_{2}=4$, at centre of membrane 